\title{
A NOTE ON THE LOG-LOGISTIC AND TRANSMUTED LOG-LOGISTIC MODELS. SOME APPLICATIONS
}

\author{
SVETOSLAV MARKOV ${ }^{1}$, NIKOLAY KYURKCHIEV ${ }^{2}$, \\ ANTON ILIEV ${ }^{3}$, AND ASEN RAHNEV 4 \\ ${ }^{1}$ Institute of Mathematics and Informatics \\ Bulgarian Academy of Sciences \\ Acad. G. Bonchev Str., Bl. 8, 1113 Sofia, BULGARIA \\ ${ }^{2,3,4}$ Faculty of Mathematics and Informatics \\ University of Plovdiv Paisii Hilendarski \\ Plovdiv, BULGARIA
}

\begin{abstract}
The Hausdorff approximation of the shifted Heaviside function by Log-logistic and quadratic transmuted Log-logistic sigmoid functions is investigated and an expression for the error of the best approximation is obtained. The results of numerical examples performed in the programming environment Mathematica confirm our theoretical conclusions. Some applications in the field of biochemical processes and debugging theory are also explored.
\end{abstract}

AMS Subject Classification: 41A46, 68N30

Key Words: log-logistic model, quadratic transmuted log-logistic model, shifted Heaviside function $h_{t_{0}}(t)$, Hausdorff approximation, upper and lower bounds, debugging theory

Received: April 13, 2018; Accepted: June 20, 2018;

Published: July 7, $2018 \quad$ doi: $10.12732 /$ dsa.v27i3.9

Dynamic Publishers, Inc., Acad. Publishers, Ltd. https://acadsol.eu/dsa

\section{INTRODUCTION}

A very good kinetic interpretation of Log-logistic dose-time response curves can be found in [5]. We will follow a brief statement from the cited article. In the context 
of kinetics mechanisms yielding Verhulst model [1]-[3] (see, also [7]-[9]), the logistic equation is defined as:

$$
\frac{d M^{*}(t)}{d t}=k M^{*}(t)\left(1-M^{*}(t)\right)
$$

where $k$ is the rate constant. The general solution is

$$
1-M^{*}(t)=\frac{1}{1+e^{k\left(t-t_{0}^{*}\right)}} .
$$

At time $t=0$ we have

$$
1-M_{0}^{*}=\frac{1}{1+e^{-k t_{0}^{*}}} ; \quad M_{0}^{*}=M^{*}(0)
$$

i.e.

$$
t_{0}^{*}=\frac{1}{k}\left(\ln \left(\frac{1}{M_{0}^{*}}-1\right)\right) .
$$

We consider the following generalization of logistic model. Let the function $M(t)$ is defined my the following nonlinear equation:

$$
\left(\frac{M}{1-M}\right)^{\frac{1}{\beta}}=1+\frac{k\left(t-t_{0}\right)}{\beta} .
$$

After differentiation of both sides of Eq. (2), and after simple calculation we get the following differential equation for the new Log-logistic function $M(t)$ :

$$
\frac{d M(t)}{d t}=k M^{1-\frac{1}{\beta}}(1-M)^{1+\frac{1}{\beta}}
$$

where $\beta$ is a shape parameter. For $\beta \rightarrow \infty$ the equation (3) reduces to equation (1). The Eq. (3) provides a parametric interpolation formula between the predictions of the logistic equation $(\beta \rightarrow \infty)$ and second order kinetics $(\beta=1)$. The equation $(2)$ can be rewritten as:

$$
1-M=\frac{1}{1+\left(1+\frac{k\left(t-t_{0}\right)}{\beta}\right)^{\beta}} .
$$

For $0<\frac{1}{\beta}$ it is possible to force $M=0$ at $t=0$ by setting $t_{0}=0$. With this condition the equation reduces to the simple form

$$
1-M=\frac{1}{1+\left(\frac{t}{\alpha}\right)^{\beta}},
$$

where the time constant, defined by $\alpha=\frac{\beta}{k}$ is a scale parameter.

The Log-logistic equation can be written as:

$$
M(t)=\frac{t^{\beta}}{\alpha^{\beta}+t^{\beta}}
$$


In this article we study the Hausdorff approximation of the shifted Heaviside function $h_{t_{0}}(t)$ by Log-logistic and quadratic transmuted Log-logistic cumulative distribution functions (see, for instance [20]).

We give a software modules within the programming environment CAS Mathematica for illustrating the results. Some applications in the field of biochemical processes and debugging theory have also explored.

\section{PRELIMINARIES}

Definition 1. [20] Let $T$ be a random variable with cumulative distribution function (c.d.f.) $C(t)$.

Then a general transmuted family (called $k$-transmuted family) is defined as:

$$
M(t)=C(t)+(1-C(t)) \sum_{i=1}^{k} \lambda_{i}(C(t))^{i}
$$

with $\lambda_{i} \in[-1,1]$ for $i=1,2, \ldots, k$ and $-k \leq \sum_{i=1}^{k} \lambda_{i}<1$.

For the quadratic transmuted family, see Shaw et Buckley [10]. Shaw et al. [10], Gupta et al. [12] study a new model which generalizes the Log-logistic function [13]. The Log-logistic distribution (also known as the Fisk distribution [11]) is a widely used lifetime distribution. For other results, see [21].

The distribution is used to model in fields such as biostatistics, population dynamic, medical research [6] and economics.

The (c.d.f.) of Log-logistic distribution is given by:

$$
C(t)=\frac{t^{\beta}}{\alpha^{\beta}+t^{\beta}}, \quad t \in[0, \infty) .
$$

Definition 2. The (c.d.f.) of quadratic transmuted Log-logistic family is defined by:

$$
M_{1}(t)=\frac{t^{\beta}}{\alpha^{\beta}+t^{\beta}}\left(1+\lambda-\lambda \frac{t^{\beta}}{\alpha^{\beta}+t^{\beta}}\right) .
$$

Remark. From (5) we have

$$
M_{1}(t)=C(t)+(1-C(t))\left(\lambda_{1} C(t)+\lambda_{2} C^{2}(t)\right)
$$


If $\lambda_{2}=0$ and $\lambda_{1}=\lambda$ we have

$$
\begin{aligned}
M_{1}(t) & =C(t)+(1-C(t)) \lambda C(t) \\
& =\frac{t^{\beta}}{\alpha^{\beta}+t^{\beta}}+\left(1-\frac{t^{\beta}}{\alpha^{\beta}+t^{\beta}}\right) \lambda \frac{t^{\beta}}{\alpha^{\beta}+t^{\beta}} \\
& =\frac{t^{\beta}}{\alpha^{\beta}+t^{\beta}}\left(1+\lambda-\lambda \frac{t^{\beta}}{\alpha^{\beta}+t^{\beta}}\right) .
\end{aligned}
$$

Definition 3. The shifted interval Heaviside function is defined as [17]:

$$
h_{t_{0}}(t)= \begin{cases}0, & \text { if } t<t_{0}, \\ {[0,1],} & \text { if } t=t_{0}, \\ 1, & \text { if } t>t_{0} .\end{cases}
$$

We will note that the determination of compulsory in area of the Software Reliability Theory components, such as confidence intervals and confidence bounds, should also be accompanied by a serious analysis of the value of the best Hausdorff approximation of the function $h_{t_{0}}(t)$ by cumulative functions of type (4) and (6) - the subject of study in the present paper.

Definition 4. [16] The Hausdorff distance (the H-distance) $\rho(f, g)$ between two interval functions $f, g$ on $\Omega \subseteq \mathbb{R}$, is the distance between their completed graphs $F(f)$ and $F(g)$ considered as closed subsets of $\Omega \times \mathbb{R}$. More precisely,

$$
\rho(f, g)=\max \left\{\sup _{A \in F(f)} \inf _{B \in F(g)}\|A-B\|, \sup _{B \in F(g)} \inf _{A \in F(f)}\|A-B\|\right\},
$$

wherein $\|$.$\| is any norm in \mathbb{R}^{2}$, e. g. the maximum norm $\|(t, x)\|=\max \{|t|,|x|\}$; hence the distance between the points $A=\left(t_{A}, x_{A}\right), B=\left(t_{B}, x_{B}\right)$ in $\mathbb{R}^{2}$ is $\|A-B\|=$ $\max \left(\left|t_{A}-t_{B}\right|,\left|x_{A}-x_{B}\right|\right)$.

\section{MAIN RESULTS}

\subsection{A NOTE ON THE LOG-LOGISTIC FAMILY (4)}

We see that $M(\alpha)=\frac{1}{2}$. The one-sided Hausdorff distance $d$ between the function $h_{\alpha}(t)$ and the function (4) satisfies the relation

$$
M(\alpha+d)=1-d
$$

The following theorem gives upper and lower bounds for $d$

Theorem 1. For the one-sided Hausdorff distance $d=d(\alpha, \beta)$ between $h_{\alpha}(t)$ and the sigmoid (4) the following inequalities hold for $\frac{\beta}{\alpha} \geq 2$ :

$$
d_{l}=\frac{1}{1+\frac{\beta}{\alpha}}<d<\frac{\ln \left(1+\frac{\beta}{\alpha}\right)}{1+\frac{\beta}{\alpha}}=d_{r} .
$$




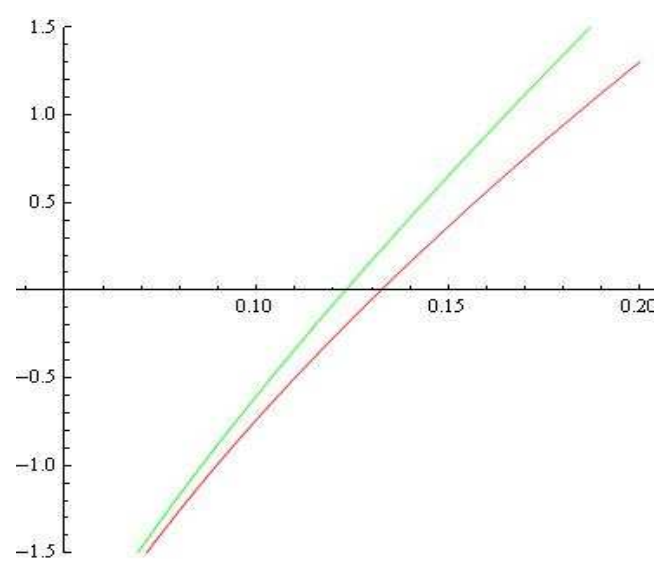

Figure 1: The functions $F(d)$ and $G(d)$.

Proof. Let us examine the function:

$$
F^{*}(d)=M(\alpha+d)-1+d
$$

From (9) we find

$$
\ln \frac{1-d}{d}=\beta \ln \frac{\alpha+d}{\alpha}=\beta \ln \left(1+\frac{d}{\alpha}\right)
$$

Consider the function

$$
F(d)=\beta \ln \left(1+\frac{d}{\alpha}\right)-\ln (1-d)-\ln \frac{1}{d} .
$$

From

$$
F^{\prime}(d)=\frac{\beta}{\alpha} \frac{1}{1+\frac{d}{\alpha}}+\frac{1}{1-d}+\frac{1}{d}>0
$$

we conclude that function $F$ is increasing.

Consider the function

$$
G(d)=\left(1+\frac{\beta}{\alpha}\right) d-\ln \frac{1}{d}
$$

From Taylor expansion we obtain $G(d)-F(d)=O\left(d^{2}\right)$.

Hence $G(d)$ approximates $F(d)$ with $d \rightarrow 0$ as $O\left(d^{2}\right)$ (see Fig. 1).

In addition $G^{\prime}(d)>0$.

Further, for $\frac{\beta}{\alpha} \geq 2$ we have

$$
G\left(\frac{1}{1+\frac{\beta}{\alpha}}\right)=1-\ln \left(1+\frac{\beta}{\alpha}\right)<0,
$$




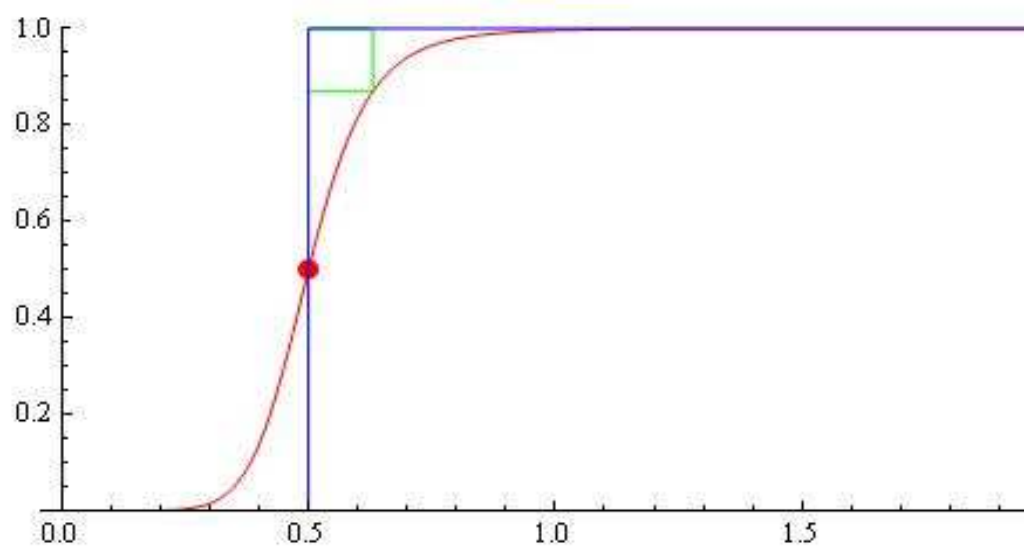

Figure 2: The model (4) for $\beta=8.2, \alpha=0.5 ; \mathrm{H}$-distance $d=0.130267$, $d_{l}=0.0574713, d_{r}=0.164165$.

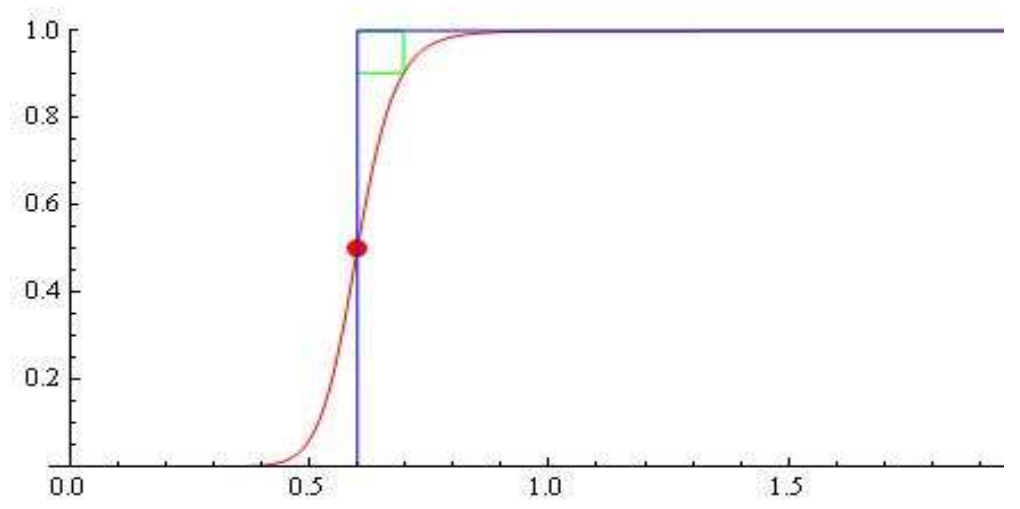

Figure 3: The model (4) for $\beta=15, \alpha=0.6$; H-distance $d=0.0964897$, $d_{l}=0.0384615, d_{r}=0.125311$.

$$
G\left(\frac{\ln \left(1+\frac{\beta}{\alpha}\right)}{1+\frac{\beta}{\alpha}}\right)=\ln \ln \left(1+\frac{\beta}{\alpha}\right)>0 .
$$

This completes the proof of the theorem.

The model (4) for $\beta=8.2, \alpha=0.5$ is visualized on Fig. 2.

From the nonlinear equation (9) and inequalities (10) we have: $d=0.130267$, $d_{l}=0.0574713, d_{r}=0.164165$.

The model (4) for $\beta=15, \alpha=0.6$ is visualized on Fig. 3.

We prove more precise bounds for $d$. Using the same notations as in Theorem 1 , 
we state the following

Theorem 2. For $\alpha, \beta \in R$

$$
\begin{aligned}
\tilde{d}_{l}= & \frac{\ln \left(1+\frac{\beta}{\alpha}\right)}{1+\frac{\beta}{\alpha}}-\frac{\ln \ln \left(1+\frac{\beta}{\alpha}\right)}{\left(1+\frac{\beta}{\alpha}\right)\left(1+\frac{1}{\ln \left(1+\frac{\beta}{\alpha}\right)}\right)}<d<\frac{\ln \left(1+\frac{\beta}{\alpha}\right)}{1+\frac{\beta}{\alpha}} \\
& +\frac{\ln \ln \left(1+\frac{\beta}{\alpha}\right)}{\left(1+\frac{\beta}{\alpha}\right)\left(\frac{\ln \ln \left(1+\frac{\beta}{\alpha}\right)}{1-\ln \left(1+\frac{\beta}{\alpha}\right)}-1\right)}=\tilde{d}_{r} .
\end{aligned}
$$

Proof. Evidently, the second derivative of (12)

$$
G^{\prime \prime}(d)=-\frac{1}{d^{2}}<0
$$

has a constant sign on $\left[\frac{1}{1+\frac{\beta}{\alpha}}, \frac{\ln \left(1+\frac{\beta}{\alpha}\right)}{1+\frac{\beta}{\alpha}}\right]$.

The straight line, defined by the points $\left(\frac{1}{1+\frac{\beta}{\alpha}}, G\left(\frac{1}{1+\frac{\beta}{\alpha}}\right)\right),\left(\frac{\ln \left(1+\frac{\beta}{\alpha}\right)}{1+\frac{\beta}{\alpha}}, G\left(\frac{\ln \left(1+\frac{\beta}{\alpha}\right)}{1+\frac{\beta}{\alpha}}\right)\right)$, and the tangent to $G(d)$ at the point $\left(\frac{\ln \left(1+\frac{\beta}{\alpha}\right)}{1+\frac{\beta}{\alpha}}, G\left(\frac{\ln \left(1+\frac{\beta}{\alpha}\right)}{1+\frac{\beta}{\alpha}}\right)\right)$, cross the abscissa at the points

$$
\begin{aligned}
& \frac{\ln \left(1+\frac{\beta}{\alpha}\right)}{1+\frac{\beta}{\alpha}}+\frac{\ln \ln \left(1+\frac{\beta}{\alpha}\right)}{\left(1+\frac{\beta}{\alpha}\right)\left(\frac{\ln \ln \left(1+\frac{\beta}{\alpha}\right)}{1-\ln \left(1+\frac{\beta}{\alpha}\right)}-1\right)}, \\
& \frac{\ln \left(1+\frac{\beta}{\alpha}\right)}{1+\frac{\beta}{\alpha}}-\frac{\ln \ln \left(1+\frac{\beta}{\alpha}\right)}{\left(1+\frac{\beta}{\alpha}\right)\left(1+\frac{1}{\ln \left(1+\frac{\beta}{\alpha}\right)}\right)},
\end{aligned}
$$

respectively.

This completes the proof of the Theorem 2 .

We note that the improved bounds (13) are more precise than (10).

\subsection{A NOTE ON THE QUADRATIC TRANSMUTED LOG-LOGISTIC CUMULATIVE SIGMOID (5)}

We consider the following family:

$$
M_{1}^{*}(t)=\frac{t^{\beta}}{\alpha^{\beta}+t^{\beta}}\left(1+\lambda-\lambda \frac{t^{\beta}}{\alpha^{\beta}+t^{\beta}}\right) .
$$

Let $t_{0}$ is the positive root of the nonlinear equation

$$
M_{1}^{*}\left(t_{0}\right)-\frac{1}{2}=0
$$

The one-sided Hausdorff distance $d_{1}$ between the function $h_{t_{0}}(t)$ and the function (14) satisfies the relation

$$
M_{1}^{*}\left(t_{0}+d_{1}\right)=1-d_{1} .
$$




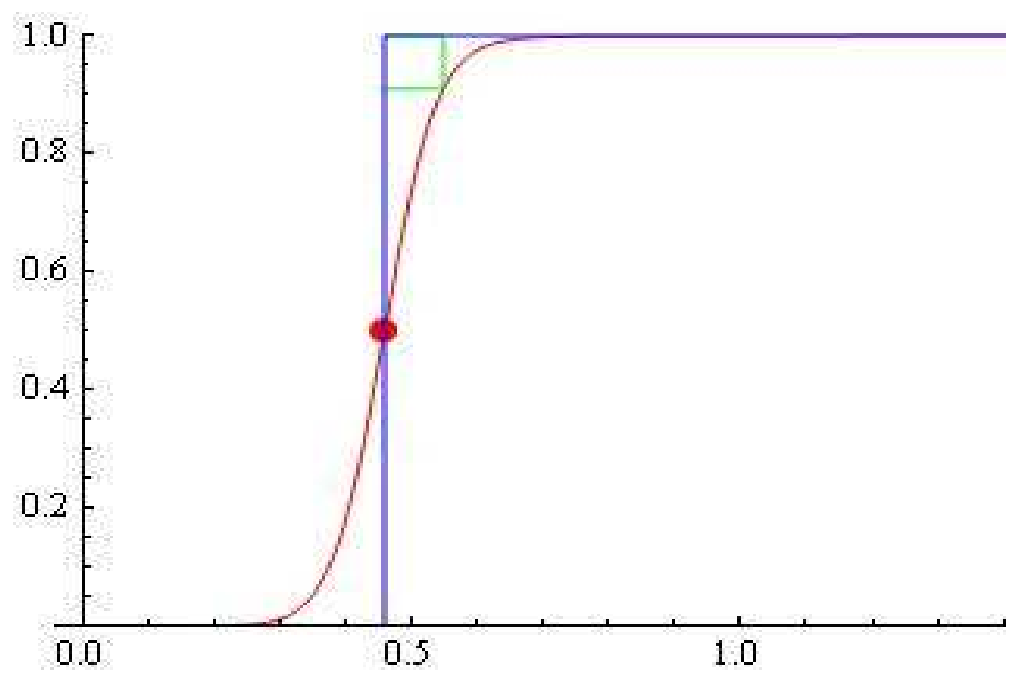

Figure 4: The model (14) for $\lambda=0.96, \alpha=0.5, \beta=10, t_{0}=0.459127$; $\mathrm{H}$-distance $d_{1}=0.0891484, d_{l_{1}}=0.0649963, d_{r_{1}}=0.177663$.

Let

$$
\begin{aligned}
& p_{1}=\frac{\alpha^{\beta}\left(-t_{0}^{\beta}-\alpha^{\beta}+\lambda t_{0}^{\beta}\right)}{\left(\alpha^{\beta}+t_{0}^{\beta}\right)^{2}}, \\
& q_{1}=1+\frac{2 \beta \lambda t_{0}^{3 \beta-1}}{\left(\alpha^{\beta}+t_{0}^{\beta}\right)^{3}}-\frac{\beta(1+3 \lambda) t_{0}^{2 \beta-1}}{\left(\alpha^{\beta}+t_{0}^{\beta}\right)^{2}}+\frac{\beta(1+\lambda) t_{0}^{\beta-1}}{\alpha^{\beta}+t_{0}^{\beta}} .
\end{aligned}
$$

The following theorem gives upper and lower bounds for $d_{1}$

Theorem 3. For the one-sided Hausdorff distance $d_{1}$ between $h_{t_{0}}(t)$ and the sigmoid (14) the following inequalities hold for:

$$
\begin{gathered}
2.1 q_{1}>e^{1.05} \\
d_{l_{1}}=\frac{1}{2.1 q_{1}}<d_{1}<\frac{\ln \left(2.1 q_{1}\right)}{2.1 q_{1}}=d_{r_{1}} .
\end{gathered}
$$

The proof follows the ideas given in this note and will be omitted.

The model (14) for $\lambda=0.96, \alpha=0.5, \beta=10, t_{0}=0.459127$ is visualized on Fig. 4. 


\section{SOME APPLICATIONS}

1. The Hill's function is used frequently to study biochemical processes in the living cell (see, e.g. [14], [15]). Hill's model is concerned with the reaction network:

$$
C+\beta T \longleftrightarrow C_{\beta}
$$

where $C$ denotes a protein that binds up to $\beta$ molecules of ligand $T$ and $C_{\beta}$ is a ligand-protein complex. The coefficient $\beta$ describes the number of binding sites on the protein.

All ligands bind simultaneously. Assuming that both the forward and backward reactions are allowed applying the mass action law we obtain for the rate of the ligand concentration a simple expression.

We may assume that this expression equals zero, because the ligand concentration is much bigger than the protein concentration and thus dot change in time. From the latter equation one easily derive the expression for the dose response curve (the Hill's function) which relates the amount of free ligands, $t$, to the fraction of ligand-bound proteins (e.g. receptors) in the system, $\varphi$.

In biochemistry, the proportion of the bound macromolecules is often described by Hill's equation [4]:

$$
\varphi=\frac{\frac{t^{\beta}}{K}}{1+\frac{t^{\beta}}{K}},
$$

where $K$ denotes the dissociation constant.

When $\beta$ is an integer, this formula can be explained by chemical kinetics. In many cases, the value of $\beta$ is not an integer. For example, for the binding of oxygen to haemoglobin, we have $\beta \approx 2.8$ [14].

In the case $K=\alpha^{\beta}$ we have the $\log -$ logistic function

$$
M(t)=\frac{t^{\beta}}{\alpha^{\beta}+t^{\beta}}
$$

The function $M(t)$ is the general Hill time-response equation

$$
1-M(t)=\frac{1}{1+\left(\frac{t}{\alpha}\right)^{\beta}}
$$

that describes the temporal transformation of the population mechanism [15].

Now it is clear how important is the study of the phenomenon "super saturation", to which we devoted the Theorems $1-2$.

2. The research of each new model in the field of debugging and test theory compulsory passes through the experimental phase with imposed in practice databases. 


\begin{tabular}{|l|l|l|l|l|}
\hline $\begin{array}{l}\text { Month In- } \\
\text { dex }\end{array}$ & $\begin{array}{l}\text { System Days } \\
(\text { Days })\end{array}$ & $\begin{array}{l}\text { System Days }(\mathrm{Cu}- \\
\text { mulative })\end{array}$ & Failures & $\begin{array}{l}\text { Cumulative } \\
\text { Failures }\end{array}$ \\
\hline 1 & 961 & 961 & 7 & 7 \\
\hline 2 & 4170 & 5131 & 3 & 10 \\
\hline 3 & 8789 & 13,920 & 14 & 24 \\
\hline 4 & 11,858 & 25,778 & 8 & 32 \\
\hline 5 & 13,110 & 38,888 & 11 & 43 \\
\hline 6 & 14,198 & 53,086 & 8 & 51 \\
\hline 7 & 14,265 & 67,351 & 7 & 58 \\
\hline 8 & 15,175 & 82,526 & 19 & 77 \\
\hline 9 & 15,376 & 97,902 & 17 & 94 \\
\hline 10 & 15,704 & 113,606 & 6 & 100 \\
\hline 11 & 18,182 & 131,788 & 11 & 111 \\
\hline 12 & 17,760 & 149,548 & 4 & 115 \\
\hline 13 & 18,352 & 167,900 & 0 & 115 \\
\hline
\end{tabular}

Table 1: Field failure data [18].

One of them is the data provided in [18]. The operating time of the software is 167,900 days. 115 failures are detected for these days which contain 71 unique failures.

Table 1 shows the failures data which are united for each of the 13 months.

Dataset included [19] Year 2000 compatibility modifications, operating system upgrade, and signaling message processing.

Below, we will illustrate the fitting of this data, for example, with the $M(t)$ model, and will show the connection to discussed in this article - approximate task.

The fitted model

$$
M(t)=N \frac{t^{\beta}}{\alpha^{\beta}+t^{\beta}}
$$

based on the data of Table 1 for the estimated parameters: $N=115 ; \alpha=5.93046 ; \beta=$ 3.15179 is plotted on Fig. 5 .

The example results show a good fit by the presented model $M(t)$.

The fitted model

$$
M_{1}(t)=N \frac{t^{\beta}}{\alpha^{\beta}+t^{\beta}}\left(1+\lambda-\lambda \frac{t^{\beta}}{\alpha^{\beta}+t^{\beta}}\right)
$$

based on the Dataset for the estimated parameters: $N=115 ; \quad \alpha=17.8094 ; \quad \beta=$ $1.71172 ; \lambda=2.92657$ is plotted on Fig. 6 .

The approximation of these data by quadratic transmuted function provides very good results. 


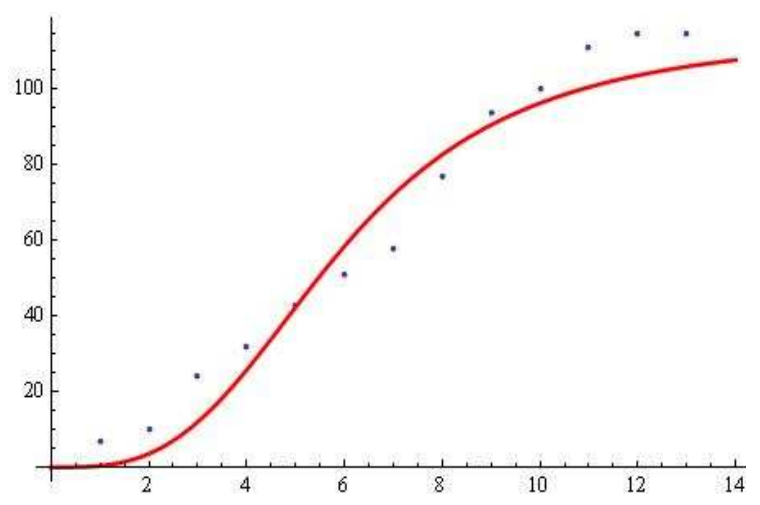

Figure 5: Approximation solution by $M(t)$.

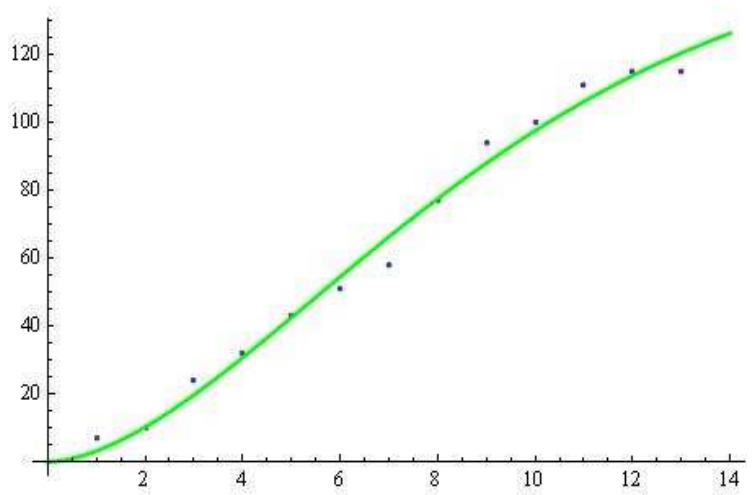

Figure 6: Approximation solution by model $M_{1}^{*}(t)$.

The given comparison shows that in some cases the quadratic transmuted Loglogistic software reliability model is better than that of Log-logistic software reliability model (see, Fig. 7).

Obviously, studying of phenomenon "super saturation" is mandatory element along with other important components - "confidence bounds" and "confidence intervals" when dealing with questions from Software Reliability Models domain.

For some software reliability models, see [22]-[39].

We hope that the results will be useful for specialists in this scientific area. 


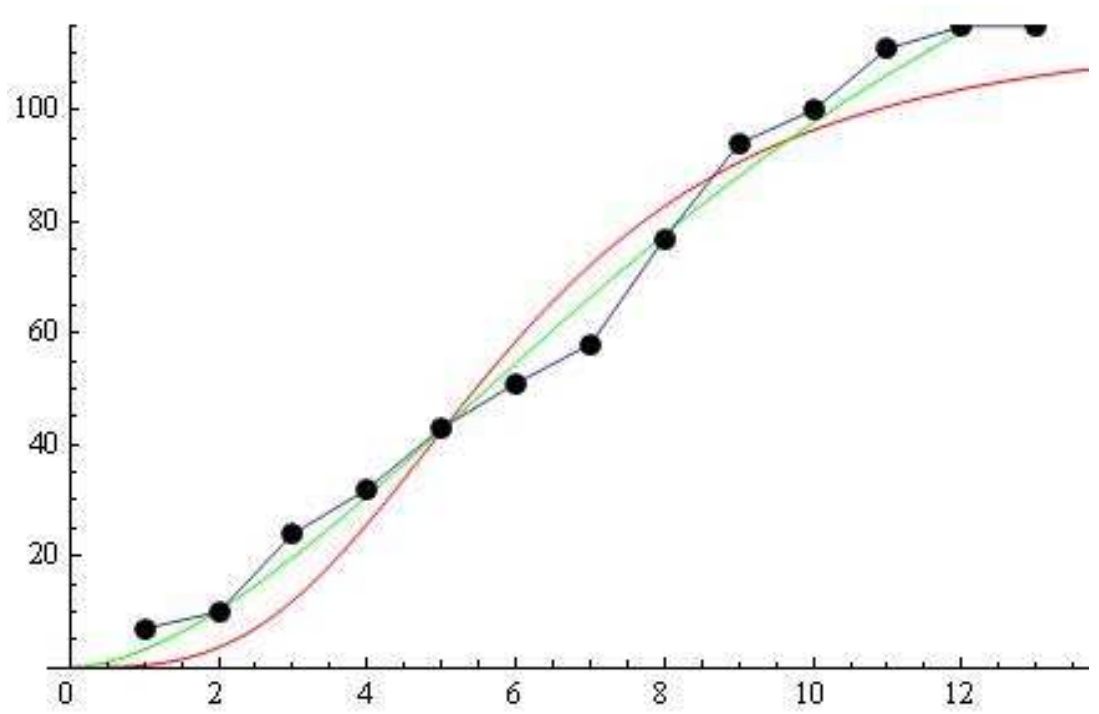

Figure 7: Comparison between Log-logistic (red) and quadratic transmuted Log-logistic (green) models.

\section{ACKNOWLEDGMENTS}

This work has been supported by the project FP17-FMI-008 of Department for Scientific Research, Paisii Hilendarski University of Plovdiv.

\section{REFERENCES}

[1] P.-F. Verhulst, Notice sur la loi que la population poursuit dans son accroissement, Correspondance Mathematique et Physique, 10 (1838), 113-121.

[2] P.-F. Verhulst, Recherches Mathematiques sur la loi d'accroissement de la population (Mathematical researches into the law of population growth increase), Nouveaux Memoires de l'Academie Royale des Sciences et Belles-Lettres de Bruxelles, 18 (1845), 1-42.

[3] P.-F. Verhulst, Deuxieme memoire sur la loi d'accroissement de la population, Memoires de l'Academie Royale des Sciences, des Lettres et des Beaux-Arts de Belgique, 20 (1847), 1-32.

[4] A.K. Hill, The possible effects of the aggregation of the molecules of haemoglobin on its dissociation curves, The Journal of Physiology, 40, IV-VII (1910). 
[5] W. Focke, I. van der Westhuizen, N. Musee, M. Loots, Kinetic interpretation of log-logistic dose-time response curves, Scientific Reports, 7, Article number:2234 (2017).

[6] D. Collet, Modelling Survival Data in Medical Research, (2nd ed.), CRC press, 2003, ISBN 1-58488-325-1.

[7] N. Kyurkchiev, S. Markov, Sigmoid Functions: Some Approximation and Modelling Aspects, LAP LAMBERT Academic Publishing, Saarbrucken, 2015, ISBN 978-3-659-76045-7.

[8] N. Kyurkchiev, S. Markov, On the Hausdorff distance between the Heaviside step function and Verhulst logistic function, J. Math. Chem., 54, No. 1 (2016), 109-119, DOI: 10.1007/S10910-015-0552-0.

[9] N. Kyurkchiev, A new transmuted cumulative distribution function based on the Verhulst logistic function with application in population dynamics, Biomath Communications, 4, No. 1 (2017).

[10] W.T. Shaw, I.R. Buckley, The alchemy of probability distributions: Beyond Gram-Charlier expansions, and skew-kurtotic-normal distribution from a rank transmutation map, 2007, UCL discovery repository; http://discovery.ucl.uk/id/ eprint/643923

[11] P.R. Fisk, The graduation of income distribution, Econometrica, 29, No. 2 (1961), 171-185.

[12] R.C. Gupta, O. Acman, S. Lvin, A study of log-logistic model in survival analysis, Biometrica Journal, 41, No. 4 (1999), 431-443.

[13] G. Aryal, Transmuted log-logistic distribution, J. of Statistics Applications and Probability, 2, No. 1 (2015), 11-20.

[14] A. Ortiz, Derivation of Hill's equation from scale invariance, Journal of Uncertain Systems, 7, No. 3 (2013), 198-202.

[15] Z. Konkoli, Safe uses of Hill's model: An exact comparison with the Adair-Klotz model, Theoretical Biology and Medical Modelling (2011).

[16] B. Sendov, Hausdorff Approximations, Kluwer, Boston, 1990.

[17] R. Anguelov, S. Markov, Hausdorff continuous interval functions and approximations, In SCAN 2014 Proceedings, LNCS, ed. by J.W.von Gudenberg, Springer, Berlin, 2015.

[18] D.R. Jeske, X. Zhang, Some successful approaches to software reliability modeling in industry, J. Syst. Softw., 74 (2005), 85-99. 
[19] K. Song, H. Pham, A Software reliability model with a weibull fault detection rate function subject to operating environments, Appl. Sci., 7 (2017), 7, 983; doi:10.3390/app7100983, 16 pp.

[20] Rahman, M., B. Al-Zahrani, M. Shahbaz, A general transmuted family of distribution, Pak. J. Stat. Oper. Res., 14, No. 2 (2018), 451-469.

[21] N. Pavlov, A. Golev, A. Iliev, A. Rahnev, N. Kyurkchiev, On the KumaraswamyDagum log-logistic sigmoid function with applications to population dynamics, Biomath Communications, 5, No. 1 (2018).

[22] J. D. Musa, A. Ianino, K. Okumoto, Software Reliability: Measurement, Prediction, Applications, McGraw-Hill, 1987.

[23] H. Pham, System Software Reliability, In: Springer Series in Reliability Engineering, Springer-Verlag London Limited, 2006.

[24] S. Yamada, Software Reliability Modeling: Fundamentals and Applications, Springer, 2014.

[25] S. Yamada, Y. Tamura, OSS reliability measurement and assessment, In: Springer Series in Reliability Engineering (H. Pham, Ed.), Springer International Publishing Switzerland, 2016.

[26] A. Pandey, N. Goyal, Early software reliability prediction. A fuzzy logic approach, In: Studies in Fuzziness and Soft Computing (J. Kacprzyk, Ed.), vol. 303, Springer, London, 2013.

[27] M. Bisi, N. Goyal, Artificial neural network for software reliability prediction, In: Performability Engineering Series (K. Misra and J. Andrews, Eds.), John Wiley \& Sons, Inc., New Jersey, 2017.

[28] P. K. Kapur, H. Pham, A. Gupta, P. C. Jha, Software reliability assessment with OR Applications, In: Springer Series in Reliability Engineering, Springer-Verlag, London, 2011.

[29] V. Ivanov, A. Reznik, G. Succi, Comparing the reliability of software systems: A case study on mobile operating systems, Information Sciences, 423 (2018), 398-411.

[30] N. Pavlov, A. Iliev, A. Rahnev, N. Kyurkchiev, Some Software Reliability Models: Approximation and Modeling Aspects, LAP LAMBERT Academic Publishing, 2018, ISBN: 978-613-9-82805-0

[31] N. Pavlov, G. Spasov, A. Rahnev, N. Kyurkchiev, A new class of Gompertz-type software reliability models, International Electronic Journal of Pure and Applied Mathematics, 12, No. 1 (2018), 43-57. 
[32] N. Pavlov, G. Spasov, A. Rahnev, N. Kyurkchiev, Some deterministic reliability growth curves for software error detection: Approximation and modeling aspects, International Journal of Pure and Applied Mathematics, 118, No. 3 (2018), 599611.

[33] N. Pavlov, A. Golev, A. Rahnev, N. Kyurkchiev, A note on the Yamada-exponential software reliability model, International Journal of Pure and Applied Mathematics, 118, No. 4 (2018), 871-882.

[34] N. Pavlov, A. Iliev, A. Rahnev, N. Kyurkchiev, A Note on the "mean value" software reliability model, International Journal of Pure and Applied Mathematics, 118, No. 4 (2018), 949-956.

[35] N. Pavlov, A. Golev, A. Rahnev, N. Kyurkchiev, A note on the generalized inverted exponential software reliability model, International Journal of Advanced Research in Computer and Communication Engineering, 7, No. 3 (2018), 484487.

[36] N. Pavlov, A. Iliev, A. Rahnev, N. Kyurkchiev, Transmuted inverse exponential software reliability model, Int. J. of Latest Research in Engineering and Technology, 4, No. 5 (2018), 1-6.

[37] N. Pavlov, A. Iliev, A. Rahnev, N. Kyurkchiev, On the extended Chen's and Pham's software reliability models. Some applications, Int. J. of Pure and Appl. Math., 118, No. 4 (2018), 1053-1067.

[38] N. Pavlov, A. Golev, A. Rahnev, N. Kyurkchiev (2018), Analysis of the Chen's and Pham's software reliability models, Cybernetics and Information Technologies, 18, No. 2 (2018), Accepted.

[39] N. Pavlov, A. Iliev, A. Rahnev, N. Kyurkchiev, On some nonstandard software reliability models, C.R. Acad. Bul. Sci. (2018), To Appear. 
